\title{
Recent advances on the mechanisms of kidney stone formation (Review)
}

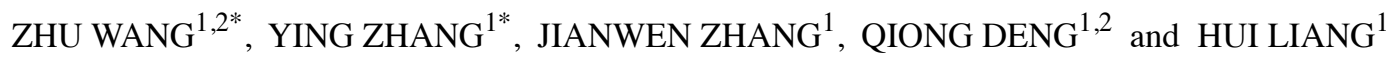 \\ ${ }^{1}$ Department of Urology and ${ }^{2}$ Central Laboratory, People's Hospital of Longhua, \\ Southern Medical University, Shenzhen, Guangdong 518109, P.R. China
}

Received April 4, 2021; Accepted May 31, 2021

DOI: $10.3892 /$ ijmm.2021.4982

\begin{abstract}
Kidney stone disease is one of the oldest diseases known to medicine; however, the mechanisms of stone formation and development remain largely unclear. Over the past decades, a variety of theories and strategies have been developed and utilized in the surgical management of kidney stones, as a result of recent technological advances. Observations from the authors and other research groups suggest that there are five entirely different main mechanisms for kidney stone formation. Urinary supersaturation and crystallization are the driving force for intrarenal crystal precipitation. Randall's plaques are recognized as the origin of calcium oxalate stone formation. Sex hormones may be key players in the development of nephrolithiasis and may thus be potential targets for new drugs to suppress kidney stone formation. The microbiome, including urease-producing bacteria, nanobacteria and intestinal microbiota, is likely to have a profound effect on urological health, both positive and negative, owing to its metabolic output and other contributions. Lastly, the immune response, and particularly macrophage differentiation, play crucial roles in renal calcium oxalate crystal formation. In the present study, the current knowledge for each of these five aspects of kidney stone formation is reviewed. This knowledge may be used to explore novel research opportunities and improve the understanding of the initiation and development of kidney stones for urologists, nephrologists and primary care.
\end{abstract}

Correspondence to: Dr Zhu Wang or Dr Hui Liang, Department of Urology, People's Hospital of Longhua, Southern Medical University, 38 Jinglong Jianshe Road, Shenzhen, Guangdong 518109, P.R. China E-mail: wangzhu1223@hotmail.com

E-mail: lianghui1976@163.com

${ }^{*}$ Contributed equally

Key words: kidney stone, urolithiasis, mechanism, nanobacteria, microbiome

\section{Contents}

\section{Introduction}

2. Physicochemical mechanism of kidney stone formation

3. Randall's plaque and calcium oxalate stone formation

4. Role of sex hormones in calcium oxalate nephrolithiasis

5. Role of the microbiome in stone formation

6. Immune response to urinary crystals

7. Conclusion and future perspectives

\section{Introduction}

Kidney stone disease, also known as nephrolithiasis or urolithiasis, is one of the oldest diseases known to medicine. It is estimated that $1-15 \%$ individuals suffer from kidney stone formation at some point during their lifetime, and the prevalence and incidence of kidney stone is reported to be increasing worldwide $(1,2)$. A recent study concluded that the prevalence of kidney stones was $5.8 \%$ among Chinese adults $(6.5 \%$ in men and $5.1 \%$ in women), with about 1 in 17 adults currently affected (3). Without proper treatment, kidney stones can cause the blockage of the ureter, blood in the urine, frequent urinary tract infections, vomiting or painful urination, culminating in the permanent functional damage of the kidneys (4). The worldwide prevalence of urolithiasis has increased over the past decades. Urolithiasis is often a recurrent and lifelong disease with a recurrence rate of 50\% within 5-10 years and $75 \%$ within 20 years (5). Some studies have indicated that an increase in kidney stone occurrence is expected, due to multiple environmental factors, including changes in lifestyle and dietary habits, as well as global warming $(1,4,6)$. However, precise factors responsible for the upward prevalence and recurrence of urolithiasis have not been identified yet. Due to its high prevalence in adults of working age, kidney stone disease has a substantial impact on the individual and society, and has become a public health issue, particularly in populations residing in regions with a hot and dry climate $(7,8)$.

There are mainly five types of kidney stones according to the mineralogical composition, including calcium oxalate (CaOx; 65.9\%), carbapatite $(15.6 \%)$, urate $(12.4 \%)$, struvite [(magnesium ammonium phosphate), 2.7\%], brushite $(1.7 \%)(9,10)$. Kidney stones can be broadly categorized into calcareous (calcium containing) stones and non-calcareous 
stones. The most common types of human kidney stones are $\mathrm{CaOx}$ and calcium phosphate $(\mathrm{CaP})$, either alone or combined, which are calcareous and radio-opaque stones $(9,11)$. Kidney stones form at a foundation of CaP termed Randall's plaques (RPs), which begins at the basement membranes of thin limbs of the loop of Henle on the renal papillary surface (12). $\mathrm{CaOx}$ and urate stones exhibit a higher occurrence in males, whereas higher percentages of carbapatite and struvite stones are observed in females than in males $(10,13)$. However, the role of sex differences in the pathophysiological mechanisms of urinary stone disease are not yet fully understood.

Regardless of the type, kidney stone formation is a complex and multistep process that includes urinary supersaturation, crystal nucleation, growth and aggregation $(11,14)$. Kidney stone formation is associated with systemic disorders, including diabetes (15), obesity, cardiovascular diseases, hypertension and metabolic syndrome $(16,17)$. Conversely, nephrolithiasis patients [also known as kidney stone formers (KSF)] are at a risk of developing hypertension (18), chronic kidney disease (CKD) (19) and progression to end-stage renal disease (ESRD) $(20,21)$. Multiple promoting factors and inhibitors have been reported to play critical roles in kidney stone formation. For example, hyperoxaluria, hyperuricosuria and phosphaturia are common promoting factors linked to kidney stone formation $(22,23)$; inter- $\alpha$-inhibitor $(\mathrm{I} \alpha \mathrm{I})$, a member of the protease inhibitor family, has been shown to inhibit $\mathrm{CaOx}$ crystallization in vitro (24).

Although details of human stone formation have accumulated, kidney stone formation and growth mechanisms are far from being clarified. The present review provides an update on the mechanisms of kidney stone formation, in order to improve the understanding of kidney stones for urologists, nephrologists and primary care givers.

\section{Physicochemical mechanism of kidney stone formation}

Urinary supersaturation and crystallization are the driving force for intrarenal crystal precipitation and is mainly caused by inherited or acquired diseases associated with renal function impairment. Additionally, urinary supersaturation and crystallization are influenced by urine $\mathrm{pH}$ and specific concentrations of substance excess, including $\mathrm{CaOx}, \mathrm{CaP}$, uric acids and urates, struvite, amino acids (cysteine), purines (2,8-dihydroxyadenine and xanthine) and drugs (e.g., atazanavir, sulfamethoxazole, amoxicillin, ceftriaxone) $(25,26)$. Additionally, crystal formation and development are influenced by multiple modulator molecules, which are known as receptors, promoters and inhibitors.

Promoters of stone formation. A number of receptors or receptor-like features have been reported to play critical roles in crystal-cell interaction, which is recognized as the most important process for crystal retention in kidney $(8,27)$. Recently, protein alterations in a $\mathrm{CaOx}$ monohydrate (COM) crystal-cell interaction model were screened by the authors, and 1,141 differentially expressed proteins (DEPs) were identified in COM treated HK-2 cells (28). Proteins and glycosaminoglycan like CD44, nucleolin, hyaluronan (HA), heat shock protein 90 (HSP90) (29), Annexin II (30) and osteopontin (OPN) $(28,31)$, have been reported to act as stone formation modulators, which has been thoroughly reviewed previously (32). Several structures and molecular components also play the role of receptor in crystal attachments, including the phosphatidylserine component of the lipid bilayer and the acidic side chains of proteins (33). Calcium, oxalate, urate and phosphate ions are the main promoters of crystal formation, which can promote crystallization of stone constituents or their aggregation through the activation of several mechanisms. Ketha et al (34) demonstrated that the first time nephrolithiasis patients had increased serum calcium and 1,25(OH)2D levels than the corresponding healthy individual serum calcium levels, suggesting that stone formation is a manifestation of altered calcium and vitamin D regulation. Higher serum calcium concentration acts as a promoter in lithogenesis, which directly regulated by the calcium-sensing receptor (CaSR) through different pathways (35). Similarly, urate and phosphate ions have also been reported to promote heterogeneous nucleation and enhance the attachment of crystals to epitheliums $(36,37)$. Another important promoter of stone formation is urine $\mathrm{pH}$ (38). Low $\mathrm{pH}$ urine may lead to $\mathrm{CaOx}$ crystallization and crystal precipitation (39). High-alkaline urine may also promote precipitation and nucleation of $\mathrm{CaOx}$ crystals $(40,41)$. Lysozyme and lactoferrin are two most recently identified proteins that promote COM growth through the acceleration of layer advancement rate on crystal surfaces (42).

Inhibitors of stone formation. Normal urine contains numerous inhibitors that act both in competition and cooperation, consequently decrease crystallization and inhibit crystals aggregationand/or adhesion to the tubularepithelialcells $(43,44)$. These inhibitors can be divided into three groups: Anions, metallic cations and macromolecules. Anions such as citrate, can inhibit crystal growth very efficiently, at concentrations above $0.1 \mathrm{mM}(45,46)$. A majority of nephrolithiasis patients exhibited a decrease in citrate excretion. Alkali supplements are widely used for hypocitraturic recurrent nephrolithiasis patients to restore citrate excretion $(47,48)$. Hydroxycitrate is a structural analog of citrate, which has been reported to show equivalent capacity in forming complexes with calcium, in order to inhibit crystallization $(49,50)$. Metallic cations such as magnesium, have been reported to inhibit crystal growth and aggregation, which is synergistic with citrate in acidic environments (51-53). Macromolecules are the most effective inhibitors of crystal growth. More specifically, OPN, Tamm-Horsfall protein (THP), urinary prothrombin fragment 1 (UPTF-1), nephrocalcin (NC) and some subunits of the serum $I \alpha I$ are able to inhibit crystal growth, aggregation and/or adhesion to the tubular cells $(11,38,45)$.

However, there is a competition between supersaturation and inhibitors of crystallization as mentioned above, which ultimately determines the pattern of crystalluria in nephrolithiasis patients and healthy individuals (54). As a consequence of the increased promoters and reduced inhibitors, crystal formation and kidney stone occurrence have been observed (Fig. 1).

\section{Randall's plaque and calcium oxalate stone formation}

RPs, first proposed by Alexander Randall in 1937 (55), are regions of subepithelial mineralized tissue at the papillary tip, 


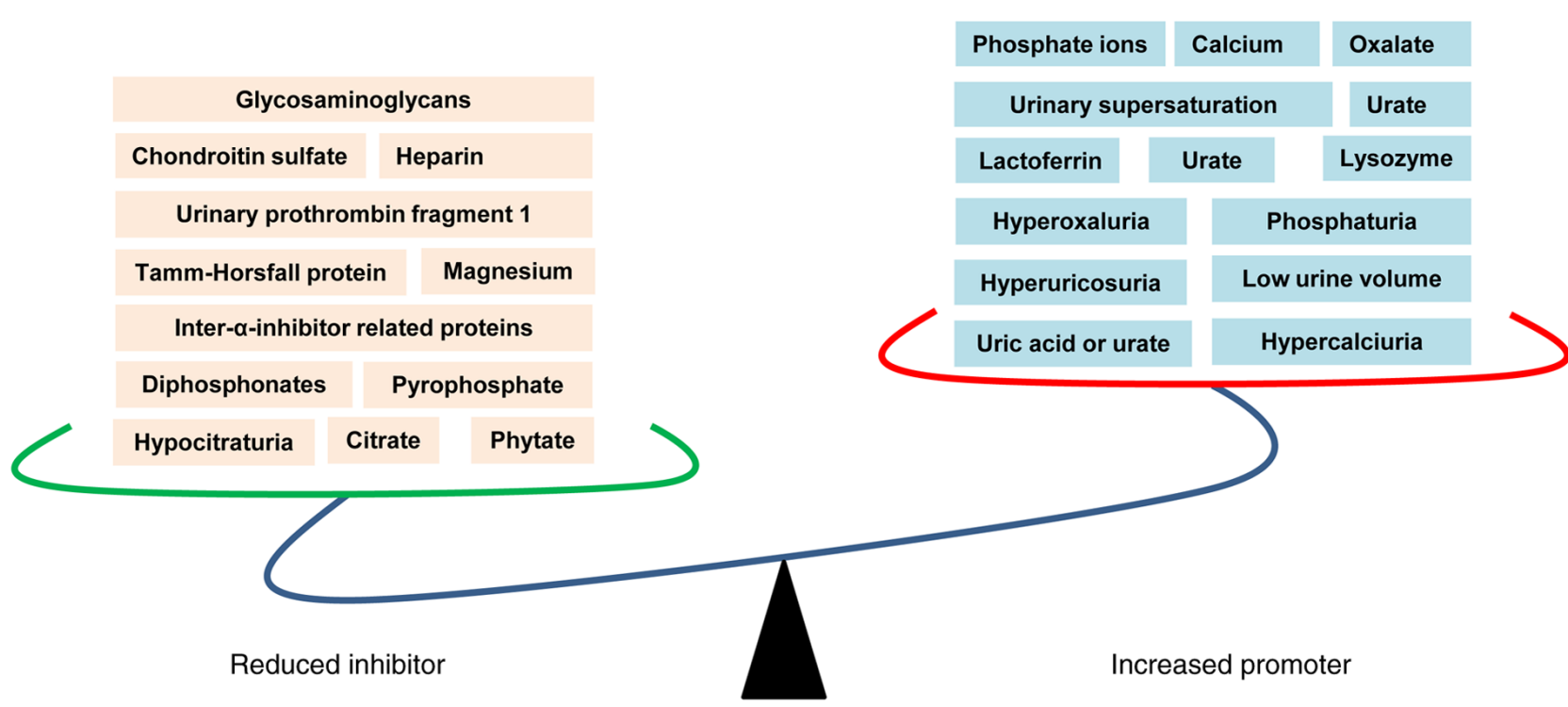

Figure 1. Physicochemical mechanisms of kidney stone formation. The reduced inhibitors (left panel) and increased promoters (right panel) are suggested to play critical roles in kidney stone formation.

surrounding the openings of the ducts of Bellini containing $\mathrm{CaP}$ (56). Scanning electron microscopy (SEM) examination has shown that RP are made of a mixing of tubules with calcified walls and of tubules obstructed by $\mathrm{CaP}$ plugs (57). $\mathrm{RP}$ consists of $\mathrm{CaP}$ crystals mixed with an organic matrix that is rich in various proteins and lipids, and includes membrane-bound vesicles or exosomes, collagen fibers, as well as other components of the extracellular matrix (58). An increasing number of studies have suggested that RPs are the origin of renal stones (57-60). Winfree et al (61) clarified that kidney stones develop as an overgrowth on RP, which contains unique organic composition (fibrillar collagen) that can be differentiated from the stone overgrowth by specific autofluorescence signatures. Of note, a previous study using a murine mode of RP revealed that vitamin D supplementation and calcium intake could notably accelerate RP formation (60). However, the precise mechanisms of RP formation remain unclear.

Recently, studies indicated that long non-coding RNAs (lcnRNAs) H19 and MALAT1 mediated osteogenic differentiation of human renal interstitial fibroblasts (hRIFs) and participated in RP formation (62-64). lcnRNA H19 has been shown to be significantly upregulated in RP, which can promote the osteogenic differentiation of hRIFs by activating Wnt/ $\beta$-catenin signaling (63). lcnRNA H19 can also serve as a facilitator in the process of $\mathrm{CaOx}$ nephrocalcinosis-induced oxidative stress and renal tubular epithelial cell injury through the interaction with miR-216b and exerts its effect via the HMGB1/TLR4/NF- $\kappa B$ signaling pathway (64). lcnRNA MALAT1 can function as a competing endogenous RNA (ceRNA) that sponges miR-320a-5p, upregulates Runx2 expression and thus promotes the osteogenic phenotype of hRIFs (62).

These studies provide novel insight into the pathogenesis of RP-mediated kidney stone disease, while further studies are urgently anticipated to explore the mechanisms of RP formation, as well as additional roles of RP in the context of stone formation.

\section{Role of sex hormones in calcium oxalate nephrolithiasis}

Statistical analyses have revealed that males have a higher incidence of $\mathrm{CaOx}$ nephrolithiasis than females at a ratio of 2-3:1 $(4,65)$; however, the exact mechanism remain unclear. Previous studies have indicated that androgens increase and estrogens decrease urinary oxalate excretion, plasma oxalate concentration and kidney $\mathrm{CaOx}$ crystal deposition. Additionally, enhanced androgen signaling may be responsible for the association between sex and kidney stone formation (65-68). Androgen receptor (AR) signaling can directly upregulate hepatic glycolate oxidase (69) and kidney epithelial nicotinamide adenine dinucleotide phosphate oxidase (NAPDH ), subunit p22-PHOX at the transcriptional level, so as to increase oxalate biosynthesis, ultimately leading to kidney stone formation (70). Peng et al (71) reported that testosterone contributes to nephrolithiasis development through the induction of renal tubular epithelial cells apoptosis and necrosis through HIF-1 $\alpha /$ BNIP3 pathway. Changtong et al (72) revealed that testosterone could promote kidney stone disease via the enhanced COM crystal-cell adhesion by the increased surface $\alpha$-enolase. Zhu et al (73) demonstrated that AR can inhibit the recruitment of macrophages and suppress the COM crystals phagocytic ability of macrophages via the decrease of the colony-stimulating factor 1 (CSF-1) signals, through miR-185-5p upregulation. These findings suggest that androgen receptor signaling may be a key player in the development of nephrolithiasis (Fig. 2).

Theoretically, AR may be a new potential target and can be evaluated for novel therapeutics for the suppression of kidney stone formation. The $5 \alpha$-reductase inhibitor, finasteride, has been reported to abolish the promoting effect of testosterone on COM crystallization (74). Another newly developed AR degradation enhancer, dimethylcurcumin (ASC-J9), has been reported to suppress oxalate crystal formation via the modulation of oxalate biosynthesis and reactive oxygen species (ROS)-induced kidney tubular epithelial cell injury in a rat model (73). Reversely, estrogen may serve as a 


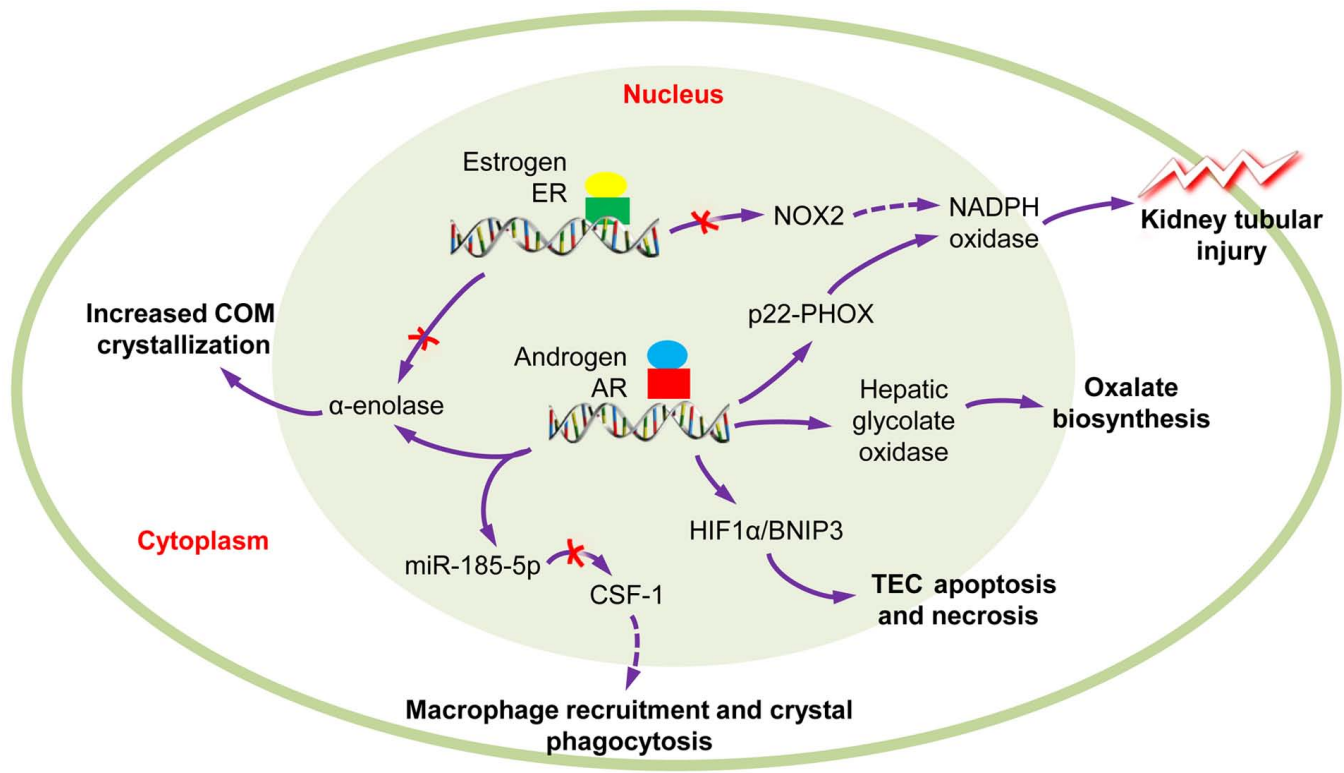

Figure 2. Role of sex hormones in calcium oxalate nephrolithiasis. The AR signaling could induce TECs apoptosis and necrosis and kidney tubular injury, promote COM crystallization and oxalate biosynthesis; however, macrophage recruitment and crystal phagocytosis are inhibited. Conversely, ER signaling can reduce ROS-mediated kidney tubular injury and COM crystallization. COM, calcium oxalate monohydrate; AR, androgen receptor; ER, estrogen receptor; ROS, reactive oxygen species.

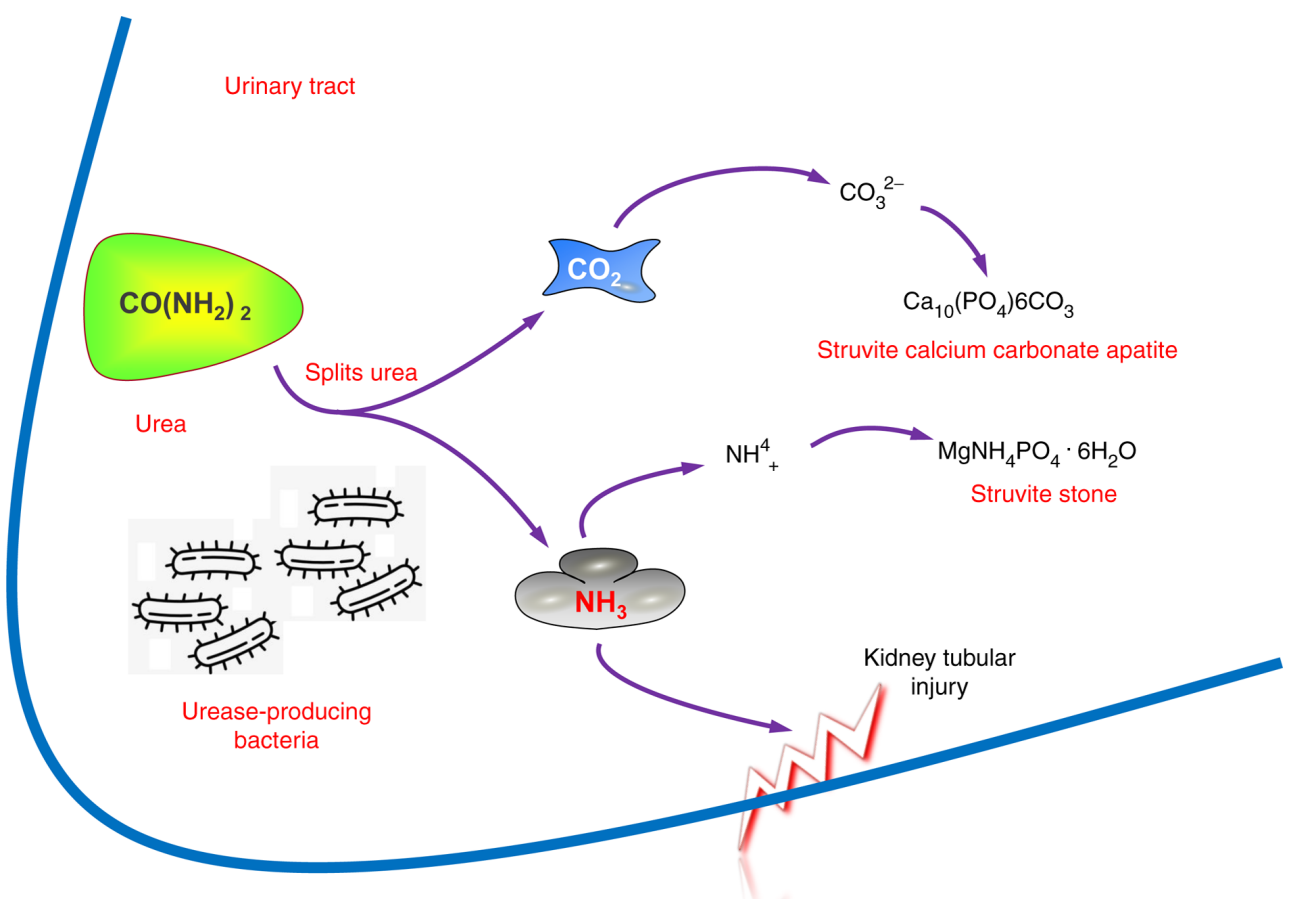

Figure 3. Role of urease-producing bacteria in stone formation. The urease-producing bacteria split urea and promote the formation of ammonia and carbon dioxide, leading to kidney tubular injury and urine alkalinization and subsequent formation of phosphate salts.

protective factor against kidney stone formation. An in vitro study demonstrated that estrogen led to changes in the cellular proteome of [Madin darby canine kidney (MDCK)] renal tubular cells that led to the decreased $\mathrm{CaOx}$ crystal receptor surface expression (annexin A1 and $\alpha$-enolase), reduced intracellular ATP, and enhanced cell proliferation and renal tubular cell tissue healing (75). There is evidence to suggest that estrogen receptor $\beta(\mathrm{ER} \beta)$ can suppress oxalate-induced oxidative stress via transcriptional suppression of the NADPH oxidase subunit 2 (NOX2) through the direct binding to the estrogen response elements (EREs) on the NOX2 5' promoter (76), which exerts protective effects on renal $\mathrm{CaOx}$ crystal deposition.

All these findings may partly explain why a higher incidence of nephrolithiasis is encounter in males than in females. Targeting AR may be developed as a potential therapy for $\mathrm{CaOx}$ crystal-related kidney stone disease. However, these studies were performed in vitro and in vivo, using only cell 


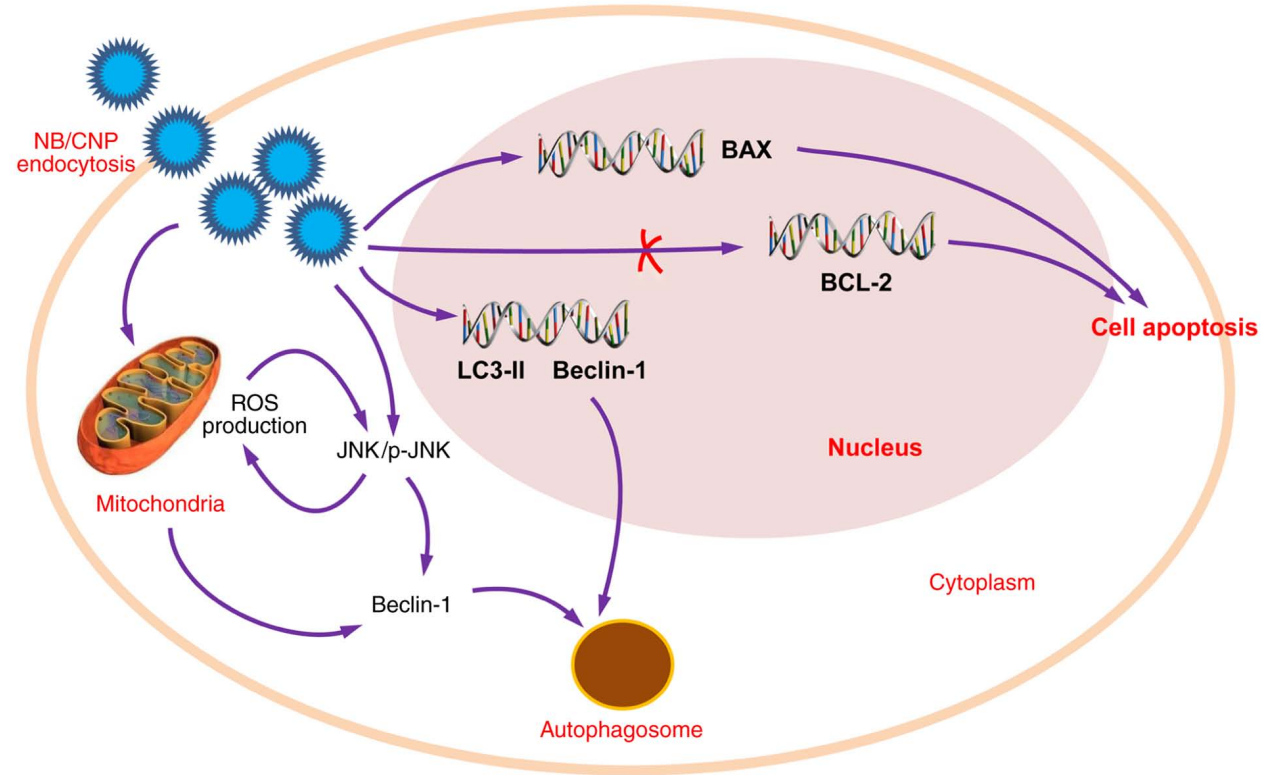

Figure 4. Role of nanobacteria in stone formation. The nanobacteria may induce ROS production through the JNK/p-JNK signaling induction, may decrease mitochondrial membrane potential and promote cell apoptosis through the downregulation of Bcl-2 expression and the upregulation of Bax expression. Additionally, nanobacteria may lead to autophagy through the upregulation of LC3-II and Beclin-1 expression. ROS, reactive oxygen species; LC3-II, microtubule-associated proteins $1 \mathrm{~A} / 1 \mathrm{~B}$ light chain $3 \mathrm{~B}$.

lines or animal models. Further validation and clinical studies are required. Finasteride and ASC-J9 have been demonstrated to suppress a number of AR-mediated diseases, including prostate cancer $(77,78)$, liver cancer and spinal and bulbar muscular atrophy neuron disease (79). However, additional future studies are necessary before the clinical application of finasteride and ASC-J9 in kidney stone prevention, considering the side-effects, including sexual dysfunction (80).

\section{Role of the microbiome in stone formation}

Emerging evidence has indicated that microorganisms belonging to the human microbiome, including microorganisms of the kidney and urinary tract, are likely to have a profound effect on urological health, both positive and negative, due to their metabolic output and other contributions (81).

Urease-producing bacteria. Urease-producing bacteria, such as Proteus mirabilis, Klebsiella pneumonia, Staphylococcus aureus, Pseudomonas aeruginosa, Providentia stuartii, Serratia and Morganella morganii, are always associated with struvite stone formation and recurrence $(82,83)$. The bacterial urease degrades urea and promotes ammonia and carbon dioxide formation, leading to urine alkalinization and phosphate salt formation (Fig. 3).

Urinary acidification and urease inhibitors have been proposed and implemented for the prevention and/or dissolution of struvite stones and encrustations in patients with infection by urea-degrading bacteria; however, their long term use is limited due to their ineffectiveness and toxicity (84). Secondarily infected stones caused by non-urease-producing bacteria, including Escherichia coli and Enterococcus spp., have also been described $(85,86)$. However, whether kidney stones form and become secondarily infected or result from a nidus of infection that propagates stone formation remains largely unclear.

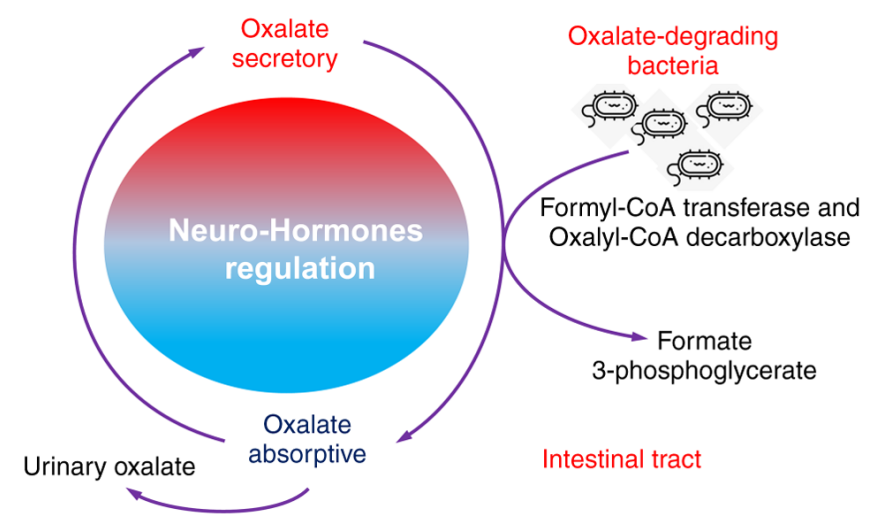

Figure 5. Role of oxalate-degrading bacteria in stone formation. Oxalate-degrading bacteria use oxalate as a carbon energy source and thrive in the presence of the oxalate anion, reduce urinary oxalate level and exhibit growth inhibition in the calcium oxalate crystallization in the kidney.

Nanobacteria $(N B)$. NB have been isolated from kidney stones for $>30$ years (87-89); however, the nature and the mechanisms involved remain obscure. Ansari et al (90) demonstrated that the size of cultured NB varies between 60 and $160 \mathrm{~nm}$, and that they could infect patients with apatite kidney stone. Kajander et al (91) indicated that NB can adapt to growing in plain DMEM or RPMI-1640, through self-proliferation. In the study by Ciftçioglu et al (92), it was demonstrated that 70 out of $72(97.2 \%)$ kidney stones contained NB. The presence of NB was independent of the stone type, although apatite-based kidney stones presented the highest immunopositivity (91). $\mathrm{NB}$ are considered to play roles in calcium nucleation, as they can produce sufficient calcium apatite in their cell walls to initiate pathologic calcifications and stone formation (93-95). This evidence is strongly in favor of the suggestion that NB are living organisms. 


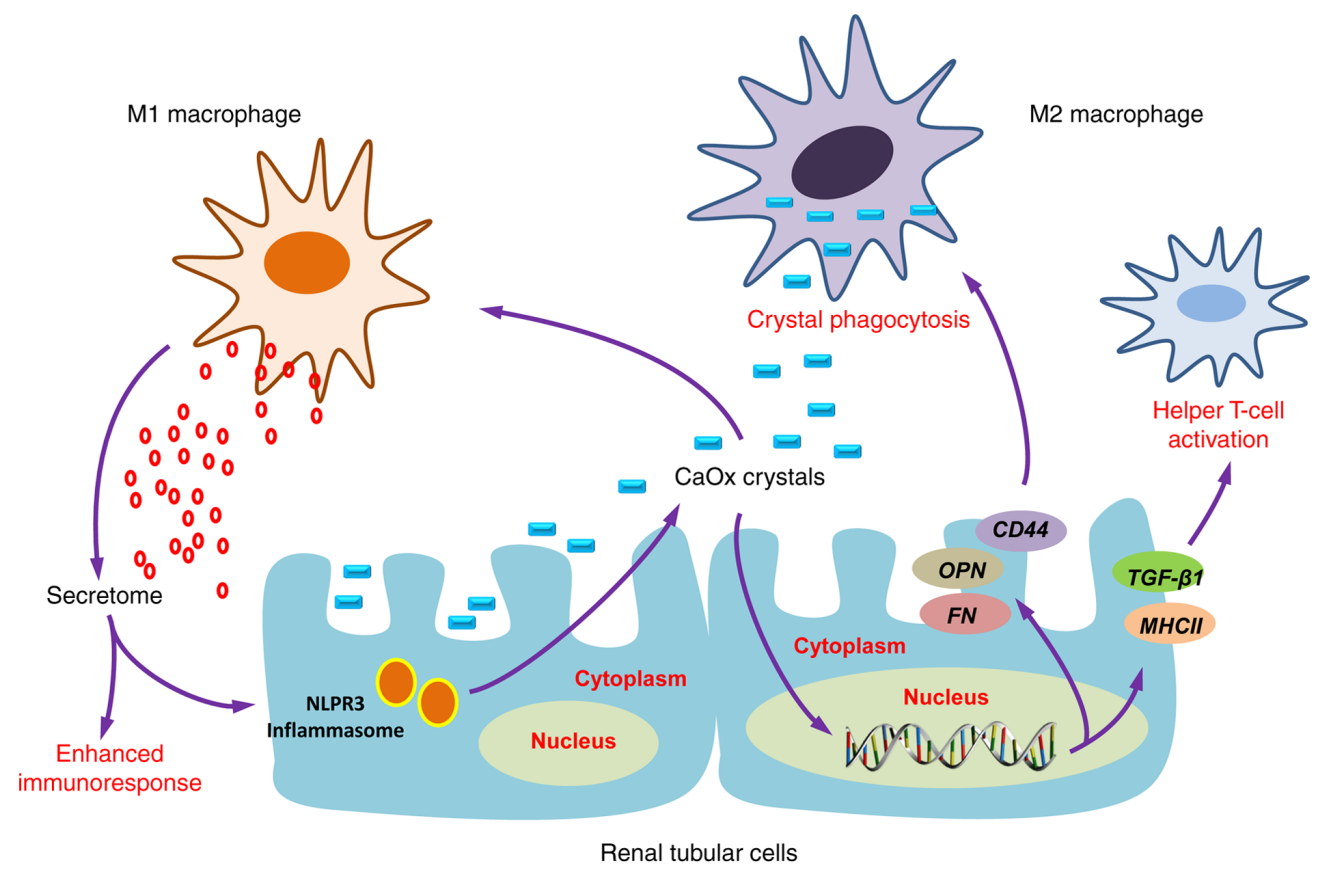

Figure 6. Immune response to urinary crystals. Macrophage accumulation and macrophage-related inflammation or anti-inflammation is the main immune response alteration observed as a result of kidney stone formation. M1 macrophages are important effectors of CaOx stone formation, while M2 macrophages could prevent $\mathrm{CaOx}$ inflammatory damage through crystal phagocytosis. $\mathrm{CaOx}$, calcium oxalate.

However, an increasing number of studies have indicated that NB, also termed 'Calcifying nanoparticles (CNPs)', 'nanobacteria-like particles' or 'Nanobes', are merely mineral protein nanoparticles with biomimetic functions $(88,89)$. Although the definition and nature of these nanoparticles remains controversial (96), their roles in kidney stone diseases has been widely reported. CNPs have been identified in RPs and have been proven to be cytotoxic to $3 \mathrm{~T} 6$ fibroblasts and HK-2 cells in vitro (89), which contributes to the renal tubular epithelial cell injury linked to kidney stone formation. Hong et al (97) demonstrated that catalase (CAT) and malonaldehyde (MDA) levels were significantly higher in CNP-treated HK-2 cells than the HK-2 control group, suggesting that CNPs may induce lipid peroxidation and result in damaging HK-2 cells. Wu et al (89) demonstrated that the CNPs may: Induce ROS production through JNK activation; decrease mitochondrial membrane potential and promote cell apoptosis through the downregulation of $\mathrm{Bcl}-2$ expression and the upregulation of Bax expression; lead to autophagy through the upregulation of microtubule-associated proteins $1 \mathrm{~A} / 1 \mathrm{~B}$ light chain 3B (LC3-II) and Beclin-1 expression (Fig. 4).

According to currently available findings in the literature, NB are localized in high concentrations in kidneys, excreted in urine, are isolated from RPs and the majority of renal stones, and play the role of the initiator, by favoring nucleation and crystal formation. Continued investigations are required, in order to solve the controversy of whether NB are living or non-living, as well as the mechanisms through which NB induce calcification and stone formation.

Intestinal microbiota. The intestinal microbiome, which has been a recent area of wide interest, has been reported to play a role in both the pathogenesis and prevention of kidney stone disease $(87,98-100)$. Oxalobacter formigenes is the most well-studied Gram-negative anaerobic bacterium that degrades oxalate in the intestinal tract and has potential probiotic characteristics for the prevention of $\mathrm{CaOx}$ kidney stone formation.

In a pilot study, Stern et al (101) investigated the distinct differences in the gut microbiome of nephrolithiasis patients, as compared with patients without kidney stone formation. Their results demonstrated that the genus Bacteroides were 3.4-fold more abundant in the kidney stone group, while the genus Prevotella were 2.8-fold more abundant in the non-stone control group. A $24 \mathrm{~h}$ urine analysis revealed that the genus Eubacterium was inversely associated with oxalate levels and the genus Escherichia trended to an inverse correlation with citrate level (101). However, the potential causative role of pre-existing dysbiosis of gut microbiome in kidney stone disease is still unclear, and the association of urinary oxalate excretion and oxalate-degrading bacteria abundances remain limited $(87,98,102,103)$.

Both absorptive and secretory pathways for oxalate have been identified in the proximal and distal segments of the colon, regulated by neuro-hormones that direct net oxalate level. Thus, it has been suggested that intestinal tract participates significantly in oxalate balance and subsequent oxalate homeostasis (104-106). The intestinal tract is also where oxalate-degrading bacteria tend to reside, particularly Oxalobacter formigenes, which requires a strict anaerobic environment to survive. One hypothesis for the role of the microbiome in the prevention of kidney stone has been that specific functional bacteria, such as the oxalate-degrading bacteria (such as Oxalobacter formigenes, Bifidobacterium sp. Porphyromonas gingivalis and Bacillus sp.) in human gut and intestinal tract, which use oxalate as their carbon energy source and thrive in the presence of the oxalate anion, exhibit growth inhibition in the $\mathrm{CaOx}$ crystallization in the kidney $(102,107,108)$ (Fig. 5). 
The activity of oxalate-degrading bacteria mediates extra-renal elimination of oxalate in the intestines and has a significantly impact on the homeostatic levels of oxalate in plasma and urine (109). This activity exhibits a strong association with the occurrence of $\mathrm{CaOx}$ stone formation.

\section{Immune response to urinary crystals}

Macrophage accumulation and macrophage-related inflammation or anti-inflammation is the main immune response alteration observed in kidney stone disease, which has been widely reported to play a crucial role in renal $\mathrm{CaOx}$ crystal formation (110).

Firstly, the recruited macrophages could promote the development of COM crystals via the interaction of CD44 with OPN and fibronectin (FN) (111), which are upregulated in renal tubular cells induced by crystals. Secondly, macrophages have been evidenced to secrete various mediators via classical secretory pathways that cause renal interstitial inflammation $(112,113)$, particularly macrophage inhibitory protein-1, monocyte chemoattractant protein-1 and interleukin-8 (IL-8) (112). These chemokines consequently enhance the recruitment of various immune cells, including monocytes, macrophages, neutrophils, dendritic cells and T-cells into the inflammatory locale $(114,115)$. Several studies have demonstrated that macrophage-derived exosomes following COM exposure are involved in kidney stone pathogenesis $(112,113,116)$. A set of proteins in COM-treated macrophage exosomes were previously identified as proteins involved mainly in immune processes, including T-cell activation and homeostasis, $\mathrm{Fc} \gamma$ receptor-mediated phagocytosis, interferon- $\gamma($ IFN- $\gamma)$ regulation and cell migration (112). Additionally, infiltrated monocytes could differentiate into different macrophage subtypes with a wide range of clinical manifestations, presentations and histological phenotypes $(110,117)$, display protective or pathogenic activities in kidney stone development (110).

Increasing evidence has revealed that M1/M2-macrophage differentiation plays an important role in renal $\mathrm{CaOx}$ crystal formation $(111,115,118-120)$. However, whether M1 macrophage-mediated inflammation that contributes to stone formation will initiate stone promoters and reduce stone inhibitors remains controversial. Khan et al (58) demonstrated that M1 macrophages could cause acute tissue injury, which was associated with crystal deposition and RP formation. Conversely, Taguchi et al (121) concluded that there was no association between renal dysfunction and increased crystal deposition, based on their observation that no changes were observed in urinary variables in lipopolysaccharide (LPS)-induced M1 macrophage-mediated acute renal injury. M2 anti-inflammatory macrophages can phagocytize and degrade $\mathrm{CaOx}$ kidney stone fragments through a clathrin-dependent mechanism $(110,113,115,120,121)$ (Fig. 6).

Given the critical role of immune-response in $\mathrm{CaOx}$ crystal formation and development, the immunotherapy approach has been proposed to prevent stone recurrences in certain individuals through the modulation of the immune response, in order to degrade $\mathrm{CaOx}$ crystals and thus prevent stones from developing (122). However, investigations into immunotherapeutic targets for kidney stone disease are urgently required.

\section{Conclusion and future perspectives}

In the present review article, emerging concepts of mechanisms contributing to stone formation were summarized, by reviewing novel insight into kidney stone disease related-metabolic risk factors, receptors, promoters and inhibitors, through the examination of the roles of immune-response, microbiome and sex hormones in stone formation and development. The pathophysiology of kidney stone disease cannot be completely explained by crystallization processes alone. However, due to current limitations in research, there are still some research areas in kidney stone formation that remain poorly understood, and were not been discussed herein. Future comprehensive studies are mandatory to further elucidate the mechanisms of the microbiome and immune response in kidney stone formation, in order to develop novel prophylactic and therapeutic approaches.

\section{Acknowledgements}

Not applicable.

\section{Funding}

The present study was supported by the National Natural Science Foundation of China (grant no. 81802566), and Shenzhen Science and Technology Program (Basic Research Project, grant no. JCYJ20180228163919346).

\section{Availability of data and materials}

Not applicable.

\section{Authors' contributions}

$\mathrm{ZW}$ and $\mathrm{YZ}$ prepared and drafted the manuscript. ZW obtained funding for the study, and drafted and revised the manuscript. QD, JZ and HL assisted in obtaining data for the review article, drafted the manuscript and provided critical revision of the manuscript for intellectual content. ZW and HL confirm the authenticity of all the raw data. All authors have read and approved the final manuscript.

\section{Ethics approval and consent to participate}

Not applicable.

\section{Patient consent for publication}

Not applicable.

\section{Competing interests}

The authors declare that they have no competing interests.

\section{References}

1. Romero V, Akpinar H and Assimos DG: Kidney stones: A global picture of prevalence, incidence, and associated risk factors. Rev Urol 12: e86-e96, 2010.

2. Morgan MS and Pearle MS: Medical management of renal stones. BMJ 352: i52, 2016. 
3. Zeng G, Mai Z, Xia S, Wang Z, Zhang K, Wang L, Long Y, Ma J, Li Y, Wan SP, et al: Prevalence of kidney stones in China: An ultrasonography based cross-sectional study. BJU Int 120 109-116, 2017.

4. Ziemba JB and Matlaga BR: Epidemiology and economics of nephrolithiasis. Investig Clin Urol 58: 299-306, 2017.

5. Eisner BH and Goldfarb DS: A nomogram for the prediction of kidney stone recurrence. J Am Soc Nephrol 25: 2685-2687, 2014

6. Brikowski TH, Lotan Y and Pearle MS: Climate-related increase in the prevalence of urolithiasis in the United States. Proc Nat Acad Sci USA 105: 9841-9846, 2008.

7. Abeywickarama B, Ralapanawa $U$ and Chandrajith $R$ : Geoenvironmental factors related to high incidence of human urinary calculi (kidney stones) in Central Highlands of Sri Lanka. Environ Geochem Health 38: 1203-1214, 2016.

8. Wang Z, Zhang JW, Zhang Y, Zhang SP, Hu QY and Liang H: Analyses of long non-coding RNA and mRNA profiling using RNA sequencing in calcium oxalate monohydrate-stimulated renal tubular epithelial cells. Urolithiasis 47: 225-234, 2019.

9. Parmar MS: Kidney stones. BMJ 328: 1420-1424, 2004

10. Ye Z, Zeng G, Yang H, Li J, Tang K, Wang G, Wang S, Yu Y, Wang Y, Zhang T, et al: The status and characteristics of urinary stone composition in China. BJU Int 125: 801-809, 2020.

11. Aggarwal KP, Narula S, KakkarM and Tandon C: Nephrolithiasis: Molecular mechanism of renal stone formation and the critica role played by modulators. Biomed Res Int 2013: 292953, 2013.

12. Khan SR, Pearle MS, Robertson WG, Gambaro G, Canales BK Doizi S, Traxer O and Tiselius HG: Kidney stones. Nat Rev Dis Primers 2: 16008, 2016.

13. Sun X, Shen L, Cong X, Zhu H, He L and Lu J: Infrared spectroscopic analysis of 5,248 urinary stones from Chinese patients presenting with the first stone episode. Urol Res 39: 339-343, 2011.

14. Hamamoto S, Taguchi K and Fujii Y: Molecular mechanism of renal stone formation. Clin Calcium 21: 1481-1487, 2011 (In Japanese).

15. Pak CY, Sakhaee K, Moe O, Preminger GM, Poindexter JR, Peterson RD, Pietrow P and Ekeruo W: Biochemical profile of stone-forming patients with diabetes mellitus. Urology 61: 523-527, 2003

16. Carbone A, Al Salhi Y, Tasca A, Palleschi G, Fuschi A, De Nunzio C, Bozzini G, Mazzaferro S and Pastore AL: Obesity and kidney stone disease: A systematic review. Minerva Urol Nefrol 70: 393-400, 2018

17. Devarajan A: Cross-talk between renal lithogenesis and atherosclerosis: An unveiled link between kidney stone formation and cardiovascular diseases. Clin Sci (Lond) 132: 615-626, 2018

18. Kittanamongkolchai W, Mara KC, Mehta RA, Vaughan LE, Denic A, Knoedler JJ, Enders FT, Lieske JC and Rule AD: Risk of hypertension among first-time symptomatic kidney stone formers. Clin J Am Soc Nephrol 12: 476-482, 2017.

19. Rule AD, Bergstralh EJ, Melton LJ III, Li X, Weaver AL and Lieske JC: Kidney stones and the risk for chronic kidney disease. Clin J Am Soc Nephrol 4: 804-811, 2009.

20. Keddis MT and Rule AD: Nephrolithiasis and loss of kidney function. Curr Opin Nephrol Hypertens 22: 390-396, 2013.

21. Dhondup T, Kittanamongkolchai W, Vaughan LE, Mehta RA, Chhina JK, Enders FT, Hickson LJ, Lieske JC and Rule AD: Risk of ESRD and mortality in kidney and bladder stone formers. Am J Kidney Dis 72: 790-797, 2018.

22. Voss S, Hesse A, Zimmermann DJ, Sauerbruch T and von Unruh GE: Intestinal oxalate absorption is higher in idiopathic calcium oxalate stone formers than in healthy controls: Measurements with the [(13)C2] oxalate absorption test. J Urol 175: 1711-1715, 2006.

23. Ha YS, Tchey DU, Kang HW, Kim YJ, Yun SJ, Lee SC and Kim WJ: Phosphaturia as a promising predictor of recurrent stone formation in patients with urolithiasis. Korean J Urol 51: $54-59,2010$.

24. Dean C, Kanellos J, Pham H, Gomes M, Oates A, Grover P and Ryall R: Effects of inter-alpha-inhibitor and several of its derivatives on calcium oxalate crystallization in vitro. Clin Sci (Lond) 98: 471-480, 2000.

25. Daudon M, Frochot V, Bazin D and Jungers P: Drug-induced kidney stones and crystalline nephropathy: Pathophysiology, prevention and treatment. Drugs 78: 163-201, 2018.

26. Rodgers AL: Physicochemical mechanisms of stone formation. Urolithiasis 45: 27-32, 2017.

27. Thongboonkerd V: Proteomics of crystal-cell interactions: A model for kidney stone research. Cells 8: 1076, 2019.
28. Wang Z, Li MX, Xu CZ, Zhang Y, Deng Q, Sun R, Hu QY, Zhang SP, Zhang JW and Liang H: Comprehensive study of altered proteomic landscape in proximal renal tubular epithelial cells in response to calcium oxalate monohydrate crystals. BMC Urol 20: 136, 2020.

29. Fong-Ngern K, Sueksakit K and Thongboonkerd V: Surface heat shock protein 90 serves as a potential receptor for calcium oxalate crystal on apical membrane of renal tubular epithelial cells. J Biol Inorg Chem 21: 463-474, 2016.

30. Kumar V, Farell G, Deganello S and Lieske JC: Annexin II is present on renal epithelial cells and binds calcium oxalate monohydrate crystals. J Am Soc Nephrol 14: 289-297, 2003.

31. Anan G, Yoneyama T, Noro D, Tobisawa Y, Hatakeyama S, Sutoh Yoneyama M, Yamamoto H, Imai A, Iwamura H, Kohada Y, et al: The impact of glycosylation of osteopontin on urinary stone formation. Int J Mol Sci 21: 93, 2019.

32. Wiener SV, Ho SP and Stoller ML: Beginnings of nephrolithiasis: Insights into the past, present and future of Randall's plaque formation research. Curr Opin Nephrol Hypertens 27: 236-242, 2018.

33. Sheng X, Ward MD and Wesson JA: Crystal surface adhesion explains the pathological activity of calcium oxalate hydrates in kidney stone formation. J Am Soc Nephrol 16: 1904-1908, 2005.

34. Ketha H, Singh RJ, Grebe SK, Bergstralh EJ, Rule AD, Lieske JC and Kumar R: Altered calcium and vitamin D homeostasis in first-time calcium kidney stone-formers. PLoS One 10: e137350, 2015.

35. Vezzoli G, Macrina L, Magni $G$ and Arcidiacono T: Calcium-sensing receptor: Evidence and hypothesis for its role in nephrolithiasis. Urolithiasis 47: 23-33, 2019.

36. Farell G, Huang E, Kim SY, Horstkorte R and Lieske JC: Modulation of proliferating renal epithelial cell affinity for calcium oxalate monohydrate crystals. J Am Soc Nephrol 15: 3052-3062, 2004

37. Gao J, Xue JF, Xu M, Gui BS, Wang FX and Ouyang JM: Nanouric acid or nanocalcium phosphate as central nidus to induce calcium oxalate stone formation: A high-resolution transmission electron microscopy study on urinary nanocrystallites. Int J Nanomedicine 9: 4399-4409, 2014

38. Ratkalkar VN and Kleinman JG: Mechanisms of stone formation. Clin Rev Bone Miner Metab 9: 187-197, 2011.

39. Moe OW, Abate N and Sakhaee K: Pathophysiology of uric acid nephrolithiasis. Endocrinol Metab Clin North Am 31: 895-914, 2002.

40. Shekarriz B and Stoller ML: Uric acid nephrolithiasis: Current concepts and controversies. J Urol 168: 1307-1314, 2002.

41. Song L and Maalouf NM: Nephrolithiasis. In: Endotext. Feingold KR, Anawalt B, Boyce A, Chrousos G, de Herder WW, Dungan K, Grossman A, Hershman JM, Hofland HJ, Kaltsas G, et al (eds). MDText.com, Inc., South Dartmouth, MA, 2000

42. Farmanesh S, Chung J, Sosa RD, Kwak JH, Karande P and Rimer JD: Natural promoters of calcium oxalate monohydrate crystallization. J Am Chem Soc 136: 12648-12657, 2014.

43. Worcester EM: Urinary calcium oxalate crystal growth inhibitors. J Am Soc Nephrol 5 (Suppl 1): S46-S53, 1994.

44. Schepers MS, van der Boom BG, Romijn JC, Schroder FH and Verkoelen CF: Urinary crystallization inhibitors do not prevent crystal binding. J Urol 167: 1844-1847, 2002.

45. Khan SR and Kok DJ: Modulators of urinary stone formation. Front Biosci 9: 1450-1482, 2004.

46. Hess B, Jordi S, Zipperle L, Ettinger E and Giovanoli R: Citrate determines calcium oxalate crystallization kinetics and crystal morphology-studies in the presence of Tamm-Horsfall protein of a healthy subject and a severely recurrent calcium stone former. Nephrol Dial Transplant 15: 366-374, 2000.

47. Cicerello E, Ciaccia M, Cova G and Mangano M: The impact of potassium citrate therapy in the natural course of Medullary Sponge Kidney with associated nephrolithiasis. Arch Ital Urol Androl 91: 102-106, 2019.

48. Siener R: Dietary treatment of metabolic acidosis in chronic kidney disease. Nutrients 10: 512, 2018.

49. Kim D, Rimer JD and Asplin JR: Hydroxycitrate: A potential new therapy for calcium urolithiasis. Urolithiasis 47: 311-320, 2019.

50. Chung J, Granja I, Taylor MG, Mpourmpakis G, Asplin JR and Rimer JD: Molecular modifiers reveal a mechanism of pathological crystal growth inhibition. Nature 536: 446-450, 2016.

51. Ryall RL, Harnett RM and Marshall VR: The effect of urine, pyrophosphate, citrate, magnesium and glycosaminoglycans on the growth and aggregation of calcium oxalate crystals in vitro. Clin Chim Acta 112: 349-356, 1981. 
52. Riley JM, Kim H, Averch TD and Kim HJ: Effect of magnesium on calcium and oxalate ion binding. J Endourol 27: 1487-1492, 2013.

53. Grases F, Rodriguez A and Costa-Bauza A: Efficacy of mixtures of magnesium, citrate and phytate as calcium oxalate crystallization inhibitors in urine. J Urol 194: 812-819, 2015.

54. Robertson WG: Do 'inhibitors of crystallisation' play any role in the prevention of kidney stones? A critique. Urolithiasis 45: 43-56, 2017.

55. Randall A: The origin and growth of renal calculi. Ann Surg 105: 1009-1027, 1937.

56. Wiener SV, Chen L, Shimotake AR, Kang M, Stoller ML and Ho SP: Novel insights into renal mineralization and stone formation through advanced imaging modalities. Connect Tissue Res 59: S102-S110, 2018

57. Daudon M, Bazin D and Letavernier E: Randall's plaque as the origin of calcium oxalate kidney stones. Urolithiasis 43 (Suppl 1): S5-S11, 2015

58. Khan SR, Canales BK and Dominguez-Gutierrez PR: Randall's plaque and calcium oxalate stone formation: Role for immunity and inflammation. Nat Rev Nephrol 17: 417-433, 2021.

59. Chung HJ: The role of Randall plaques on kidney stone formation. Transl Androl Urol 3: 251-254, 2014.

60. Bouderlique E, Tang E, Perez J, Coudert A, Bazin D, Verpont MC, Duranton C, Rubera I, Haymann JP, Leftheriotis G, et al: Vitamin D and calcium supplementation accelerates Randall's plaque formation in a murine model. Am J Pathol 189: 2171-2180, 2019.

61. Winfree S, Weiler C, Bledsoe SB, Gardner T, Sommer AJ, Evan AP, Lingeman JE, Krambeck AE, Worcester EM, El-Achkar TM and Williams JC Jr: Multimodal imaging reveals a unique autofluorescence signature of Randall's plaque. Urolithiasis 49: 123-135, 2021.

62. Zhu Z, Huang F, Xia W, Zeng H, Gao M, Li Y, Zeng F, He C, Chen J, Chen Z, et al: Osteogenic differentiation of renal interstitial fibroblasts promoted by lncRNA MALAT1 may partially contribute to Randall's plaque formation. Front Cell Dev Biol 8 : 596363, 2020.

63. Zhu Z, Cui Y, Huang F, Zeng H, Xia W, Zeng F, He C, Chen J, Chen Z, Chen $\mathrm{H}$ and Li Y: Long non-coding RNA H19 promotes osteogenic differentiation of renal interstitial fibroblasts through Wnt-beta-catenin pathway. Mol Cell Biochem 470: 145-155, 2020

64. Liu H, Ye T, Yang X, Liu J, Jiang K, Lu H, Xia D, Peng E, Chen Z, Sun F, et al: H19 promote calcium oxalate nephrocalcinosis-induced renal tubular epithelial cell injury via a ceRNA pathway. EBioMedicine 50: 366-378, 2019.

65. Fan J, Chandhoke PS and Grampsas SA: Role of sex hormones in experimental calcium oxalate nephrolithiasis. J Am Soc Nephrol 10 (Suppl 14): S376-S380, 1999.

66. Li JY, Zhou T, Gao X, Xu C, Sun Y, Peng Y, Chang Z, Zhang Y, Jiang J, Wang L and Hou J: Testosterone and androgen receptor in human nephrolithiasis. J Urol 184: 2360-2363, 2010.

67. Gupta K, Gill GS and Mahajan R: Possible role of elevated serum testosterone in pathogenesis of renal stone formation. Int J Appl Basic Med Res 6: 241-244, 2016.

68. Fuster DG, Morard GA, Schneider L, Mattmann C, Lüthi D, Vogt $B$ and Dhayat NA: Association of urinary sex steroid hormones with urinary calcium, oxalate and citrate excretion in kidney stone formers. Nephrol Dial Transplant: Dec 9, 2020 (Epub ahead of print).

69. Yoshihara H, Yamaguchi S and Yachiku S: Effect of sex hormones on oxalate-synthesizing enzymes in male and female rat livers. J Urol 161: 668-673, 1999.

70. Liang L, Li L, Tian J, Lee SO, Dang Q, Huang CK, Yeh S, Erturk E, Bushinsky D, Chang LS, et al: Androgen receptor enhances kidney stone-CaOx crystal formation via modulation of oxalate biosynthesis \& oxidative stress. Mol Endocrinol 28 . 1291-1303, 2014.

71. Peng Y, Fang Z, Liu M, Wang Z, Li L, Ming S, Lu C, Dong H Zhang W, Wang Q, et al: Testosterone induces renal tubular epithelial cell death through the HIF-1alpha/BNIP3 pathway. J Transl Med 17: 62, 2019.

72. Changtong C, Peerapen P, Khamchun S, Fong-Ngern K Chutipongtanate $\mathrm{S}$ and Thongboonkerd V: In vitro evidence of the promoting effect of testosterone in kidney stone disease: A proteomics approach and functional validation. J Proteomics 144 $11-22,2016$.

73. Zhu W, Zhao Z, Chou F, Zuo L, Liu T, Yeh S, Bushinsky D, Zeng $\mathrm{G}$ and Chang C: Loss of the androgen receptor suppresses intrarenal calcium oxalate crystals deposition via altering macrophage recruitment/M2 polarization with change of the miR-185-5p/CSF-1 signals. Cell Death Dis 10: 275, 2019.
74. Sueksakit K and Thongboonkerd V: Protective effects of finasteride against testosterone-induced calcium oxalate crystallization and crystal-cell adhesion. J Biol Inorg Chem 24: 973-983, 2019.

75. Peerapen $P$ and Thongboonkerd V: Protective cellular mechanism of estrogen against kidney stone formation: A proteomics approach and functional validation. Proteomics 19: e1900095, 2019.

76. Zhu W, Zhao Z, Chou FJ, Zuo L, Liu T, Bushinsky D, Chang C, Zeng $\mathrm{G}$ and Yeh S: The protective roles of estrogen receptor $\beta$ in renal calcium oxalate crystal formation via reducing the liver oxalate biosynthesis and renal oxidative stress-mediated cell injury. Oxid Med Cell Longev 2019: 5305014, 2019.

77. Loughlin KR: The clinical applications of five-alpha reductase inhibitors. Can J Urol 28: 10584-10588, 2021.

78. Tian H, Chou FJ, Tian J, Zhang Y, You B, Huang CP, Yeh S, Niu Y and Chang C: ASC-J9 ${ }^{\circledR}$ suppresses prostate cancer cell proliferation and invasion via altering the ATF3-PTK2 signaling. J Exp Clin Cancer Res 40: 3, 2021.

79. $\mathrm{Hu} \mathrm{H}$, Zhou $\mathrm{H}$ and $\mathrm{Xu} \mathrm{D:} \mathrm{A} \mathrm{review} \mathrm{of} \mathrm{the} \mathrm{effects} \mathrm{and} \mathrm{molecular}$ mechanisms of dimethylcurcumin (ASC-J9) on androgen receptor-related diseases. Chem Biol Drug Des 97: 821-835, 2021.

80. Andy G, John M, Mirna S, Rachita D, Michael K, Maja K, Aseem S and Zeljana B: Controversies in the treatment of androgenetic alopecia: The history of finasteride. Dermatol Ther 32: e12647, 2019.

81. Whiteside SA, Razvi H, Dave S, Reid G and Burton JP: The microbiome of the urinary tract-a role beyond infection. Nat Rev Urol 12: 81-90, 2015.

82. Bichler KH, Eipper E, Naber K, Braun V, Zimmermann R and Lahme S: Urinary infection stones. Int J Antimicrob Agents 19: 488-498, 2002.

83. Espinosa-Ortiz EJ, Eisner BH, Lange D and Gerlach R: Current insights into the mechanisms and management of infection stones. Nat Rev Urol 16: 35-53, 2019.

84. Marien T and Miller NL: Treatment of the Infected Stone. Urol Clin North Am 42: 459-472, 2015

85. de Cógáin MR, Lieske JC, Vrtiska TJ, Tosh PK and Krambeck AE: Secondarily infected nonstruvite urolithiasis: A prospective evaluation. Urology 84: 1295-1300, 2014.

86. Flannigan R, Choy WH, Chew B and Lange D: Renal struvite stones-pathogenesis, microbiology, and management strategies. Nat Rev Urol 11: 333-341, 2014.

87. Mehta M, Goldfarb DS and Nazzal L: The role of the microbiome in kidney stone formation. Int J Surg 36: 607-612, 2016.

88. Martel J, Peng HH, Young D, Wu CY and Young JD: Of nanobacteria, nanoparticles, biofilms and their role in health and disease: Facts, fancy and future. Nanomedicine (Lond) 9: 483-499, 2014.

89. Wu J, Tao Z, Deng Y, Liu Q, Liu Y, Guan X and Wang X: Calcifying nanoparticles induce cytotoxicity mediated by ROS-JNK signaling pathways. Urolithiasis 47: 125-135, 2019.

90. Ansari H, Akhavan Sepahi A and Akhavan Sepahi M: Different approaches to detect 'Nanobacteria' in patients with kidney stones: An infectious cause or a subset of life? Urol J 14: 5001-5007, 2017.

91. Kajander EO, Ciftcioglu N, Aho K and Garcia-Cuerpo E: Characteristics of nanobacteria and their possible role in stone formation. Urol Res 31: 47-54, 2003.

92. Ciftçioglu N, Björklund M, Kuorikoski K, Bergström K and Kajander EO: Nanobacteria: An infectious cause for kidney stone formation. Kidney Int 56: 1893-1898, 1999.

93. Khullar M, Sharma SK, Singh SK, Bajwa P, Shiekh FA, Relan V and Sharma M: Morphological and immunological characteristics of nanobacteria from human renal stones of a north Indian population. Urol Res 32: 190-195, 2004.

94. Shiekh FA, Khullar M and Singh SK: Lithogenesis: Induction of renal calcifications by nanobacteria. Urol Res 34: 53-57, 2006.

95. Kajander EO and Ciftçioglu N: Nanobacteria: An alternative mechanism for pathogenic intra- and extracellular calcification and stone formation. Proc Natl Acad Sci USA 95: 8274-8279, 1998

96. Abrol N, Panda A, Kekre NS and Devasia A: Nanobacteria in the pathogenesis of urolithiasis: Myth or reality? Indian J Urol 31: 3-7, 2015 .

97. Hong X, Wang X, Wang T, Yu C and Li H: Role of nanobacteria in the pathogenesis of kidney stone formation. Am J Transl Res 8: 3227-3234, 2016

98. Sadaf H, Raza SI and Hassan SW: Role of gut microbiota against calcium oxalate. Microb Pathog 109: 287-291, 2017.

99. Ticinesi A, Nouvenne A, Chiussi G, Castaldo G, Guerra A and Meschi T: Calcium oxalate nephrolithiasis and gut microbiota: Not just a gut-kidney axis. A nutritional perspective. Nutrients 12 : 548,2020 . 
100. Ticinesi A, Milani C, Guerra A, Allegri F, Lauretani F, Nouvenne A, Mancabelli L, Lugli GA, Turroni F, Duranti S, et al: Understanding the gut-kidney axis in nephrolithiasis: An analysis of the gut microbiota composition and functionality of stone formers. Gut 67: 2097-2106, 2018.

101. Stern JM, Moazami S, Qiu Y, Kurland I, Chen Z, Agalliu I, Burk R and Davies KP: Evidence for a distinct gut microbiome in kidney stone formers compared to non-stone formers. Urolithiasis 44: 399-407, 2016.

102. Falony G: Beyond Oxalobacter: The gut microbiota and kidney stone formation. Gut 67: 2078-2079, 2018.

103. Miller AW and Dearing D: The metabolic and ecological interactions of oxalate-degrading bacteria in the Mammalian gut. Pathogens 2: 636-652, 2013.

104. Worcester EM, Fellner SK, Nakagawa Y and Coe FL: Effect of renal transplantation on serum oxalate and urinary oxalate excretion. Nephron 67: 414-418, 1994.

105. Hatch M, Freel RW and Vaziri ND: Mechanisms of oxalate absorption and secretion across the rabbit distal colon. Pflugers Arch 426: 101-109, 1994

106. Peck AB, Canales BK and Nguyen CQ: Oxalate-degrading microorganisms or oxalate-degrading enzymes: Which is the future therapy for enzymatic dissolution of calcium-oxalate uroliths in recurrent stone disease? Urolithiasis 44: 45-50, 2016.

107. Knight J, Deora R, Assimos DG and Holmes RP: The genetic composition of Oxalobacter formigenes and its relationship to colonization and calcium oxalate stone disease. Urolithiasis 41: 187-196, 2013

108. Batagello CA, Monga $\mathrm{M}$ and Miller AW: Calcium oxalate urolithiasis: A case of missing microbes? J Endourol 32: 995-1005, 2018.

109. Cornelius JG and Peck AB: Colonization of the neonatal rat intestinal tract from environmental exposure to the anaerobic bacterium Oxalobacter formigenes. J Med Microbiol 53: 249-254, 2004.

110. Nikolic-Paterson DJ, Wang S and Lan HY: Macrophages promote renal fibrosis through direct and indirect mechanisms. Kidney Int Suppl (2011) 4: 34-38, 2014

111. Okada A, Yasui T, Fujii Y, Niimi K, Hamamoto S, Hirose M, Kojima Y, Itoh Y, Tozawa K, Hayashi Y and Kohri K: Renal macrophage migration and crystal phagocytosis via inflammatory-related gene expression during kidney stone formation and elimination in mice: Detection by association analysis of stone-related gene expression and microstructural observation. J Bone Miner Res 25: 2701-2711, 2010.
112. Singhto N, Kanlaya R, Nilnumkhum A and Thongboonkerd V: Roles of macrophage exosomes in immune response to calcium oxalate monohydrate crystals. Front Immunol 9: 316, 2018.

113. Singhto N and Thongboonkerd V: Exosomes derived from calcium oxalate-exposed macrophages enhance IL- 8 production from renal cells, neutrophil migration and crystal invasion through extracellular matrix. J Proteomics 185: 64-76, 2018.

114. Tamura M, Aizawa R, Hori M and Ozaki H: Progressive renal dysfunction and macrophage infiltration in interstitial fibrosis in an adenine-induced tubulointerstitial nephritis mouse model. Histochem Cell Biol 131: 483-490, 2009.

115. Kusmartsev S, Dominguez-Gutierrez PR, Canales BK, Bird VG, Vieweg $\mathrm{J}$ and Khan SR: Calcium oxalate stone fragment and crystal phagocytosis by human macrophages. J Urol 195: 1143-1151, 2016.

116. Sintiprungrat K, Singhto $\mathrm{N}$ and Thongboonkerd V: Characterization of calcium oxalate crystal-induced changes in the secretome of U937 human monocytes. Mol Biosyst 12: 879-889, 2016

117. Histiocytosis syndromes in children. Writing Group of the Histiocyte Society. Lancet 1: 208-209, 1987.

118. Okada A, Yasui T, Hamamoto S, Hirose M, Kubota Y, Itoh Y, Tozawa K, Hayashi Y and Kohri K: Genome-wide analysis of genes related to kidney stone formation and elimination in the calcium oxalate nephrolithiasis model mouse: Detection of stone-preventive factors and involvement of macrophage activity. J Bone Miner Res 24: 908-924, 2009.

119. Vervaet BA, Verhulst A, Dauwe SE, De Broe ME and D'Haese PC: An active renal crystal clearance mechanism in rat and man. Kidney Int 75: 41-51, 2009.

120. Dominguez-Gutierrez PR, Kusmartsev S, Canales BK and Khan SR: Calcium oxalate differentiates human monocytes into inflammatory M1 macrophages. Front Immunol 9: 1863, 2018.

121. Taguchi K, Okada A, Hamamoto S, Unno R, Moritoki Y, Ando R, Mizuno K, Tozawa K, Kohri K and Yasui T: M1/M2-macrophage phenotypes regulate renal calcium oxalate crystal development. Sci Rep 6: 35167, 2016.

122. Dominguez-Gutierrez PR, Kwenda EP, Khan SR and Canales BK: Immunotherapy for stone disease. Curr Opin Urol 30: 183-189, 2020 .

This work is licensed under a Creative Commons Attribution-NonCommercial-NoDerivatives 4.0 International (CC BY-NC-ND 4.0) License. 